\title{
ESTUDIO PALINOLÓGICO DEL GÉNERO SCROPHULARIA L. EN LA PENÍNSULA IBÉRICA E ISLAS BALEARES
}

\author{
Ana ORTEGA OLIVENCIA y Juan A. DEVESA ALCARAZ
}

\begin{abstract}
RESUMEN: Se estudian al microscopio óptico y electrónico de barrido los caracteres polínicos de 78 poblaciones pertenecientes a 29 táxones del género Scrophularia L. representados en la Península Ibérica e Islas Baleares, corroborándose la estenopalinidad ya conocida del género. Aunque en líneas generales la forma y tamaño de los granos carecen de interés taxonómico sí la posee, por el contrario, la densidad del retículo, que resultó de utilidad en la segregación de los táxones de las secciones Scrophularia y Canina G.Don presentes en el territorio.
\end{abstract}

Palabras clave: Scrophularia, Scrophulariaceae, polen, Península Ibérica, Islas Baleares.

ABSTRACT: Pollen grain characters of 78 populations belonging to 29 taxa of genus Scrophularia L. from the Iberian Peninsula and Balearic Islands were studied using a light and scanning electron microscopy, all of them showing a remarkable uniformity. Although the shape and size of the pollen grains in the genus are not in general important from the taxonomic point of view, however the density of the reticulum is very useful in the segregation of taxa of sections Scrophularia and Canina G.Don present in the area of study.

Key words: Scrophularia, Scrophulariaceae, pollen, Iberian Peninsula, Balearic Islands.

\section{INTRODUCCIÓN}

El género Scrophularia L. comprende alrededor de 267 táxones con distribución fundamentalmente holártica (Ortega, 1989), de los que en la Península Ibérica e Islas Baleares se encuentran representados 29, buena parte de ellos endémicos (Ortega, 1989) y pertenecientes a 2 secciones: Scrophularia (23 táxones) y Canina G.Don (6 táxones).

Aunque desde el punto de vista taxonómico el género ha sido objeto de diversas aportaciones (Stiefelhagen, 1910; Komarov, 1955; Shaw, 1962; Lall y Mill, 1978; Dalgaard, 1979; Grau, 1979 y 1981; Qaiser, 1982; Qaiser et al., 1988), los estudios palinológicos son escasos, ya que la mayor parte de las veces han abordado la familia Scrophulariaceae en su conjunto (Mohl, 1835; Risch, 1939; Hassall, 1842; Erdtman eT al., 1961; Erdtman, 1971; Varghese, 1969; Faegri y Iversen, 1975; Inceoglu y Karamustafa, 1977; Moore y Webb, 1978) o centrado sólo en algunos táxones en 
particular (Hayden, 1930; Erdtman, 1943; Straka, 1975; Verbeek-Reuvers, 1976; Qaiser $e T$ al., 1988).

En la Península Ibérica destacan las aportaciones de Pla Dalmau (1957) sobre Scrophularia canina en el NE de España, y sobre todo la de Godoy y Díez (1987) quienes realizan el estudio polínico en las 7 especies con representación en el SW de España, reconociendo únicamente el Tipo Scrophularia canina, en el que reúnen además representantes de géneros como Antirrhinum, Cymbalaria, Digitalis y Linaria.

Con el presente artículo se pretende aportar información palinológica relativa a todos los táxones representados en la Península Ibérica e Islas Baleares, que han sido recientemente revisados en sus aspectos taxonómico (Ortega, 1989; Ortega y Devesa, 1990 a; Ortega y Devesa, 1991), cariológico (Ortega y Devesa, 1990 b) y de la biología reproductiva (Ortega, 1989).

\section{MATERIAL Y MÉTODOS}

Se han estudiado muestras de 78 poblaciones correspondientes a 29 táxones (véase Anexo I), que fueron previamente acetolizadas según el método de Erdtman (1960) modificado por Hideux (1972) y montadas en glicerogelatina para su observación al microscopio óptico (MO). Para la observación al microscopio electrónico de barrido (MEB) se utilizaron igualmente granos previamente acetolizados, que fueron posteriormente sombreados con oro-paladio en alto vacío.

Siempre que fue posible se estudiaron 3 poblaciones de cada taxon, analizándose un total de 20 granos por muestra, número más que suficiente según el test de comparación de medias.

Los principales parámetros polínicos estudiados al MO fueron $\mathrm{P}$ (longitud del eje polar en corte óptico meridiano), E (longitud del eje ecuatorial en corte óptico meridiano), A (longitud de la apocolpia en corte óptico ecuatorial), M (longitud de la mesocolpia en corte óptico ecuatorial), Ex (anchura de la exina en la mesocolpia), C (anchura del colpo en corte óptico ecuatorial), Pr (anchura del poro en corte óptico meridiano) y L (anchura de los lúmenes en la mesocolpia) (véase Fig. 1). La terminología empleada para su descripción ha sido básicamente la de Erdtman (1969 y 1971) y Faegri e Iversen (1975).

Con los datos obtenidos (véase Tabla 1) se ha elaborado un diagrama de dispersión simbólica (Fig. 2), una recta de regresión en función del tamaño de la corola (Fig. 3, Tabla 2), una tabla de correlaciones entre las 9 variables estudiadas (Tabla 3) y un Análisis de Componentes Principales BMDP4 (Factorial Analysis).
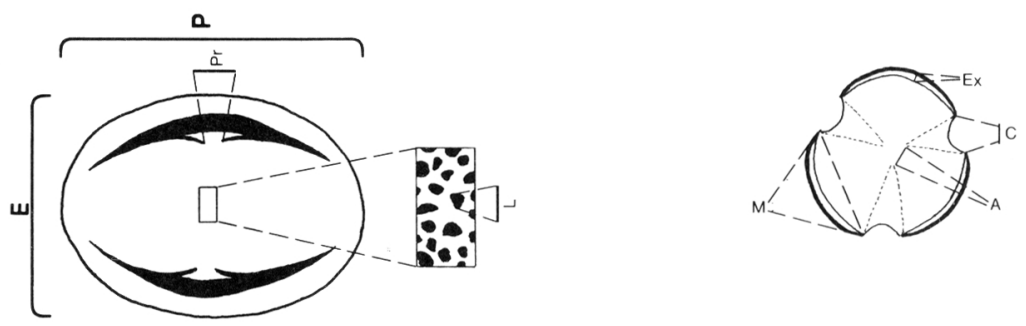

Fig. 1. Representación de un grano de polen de Scrophularia en corte óptico meridiano y en corte óptico ecuatorial, con indicación de los parámetros estudiados. 


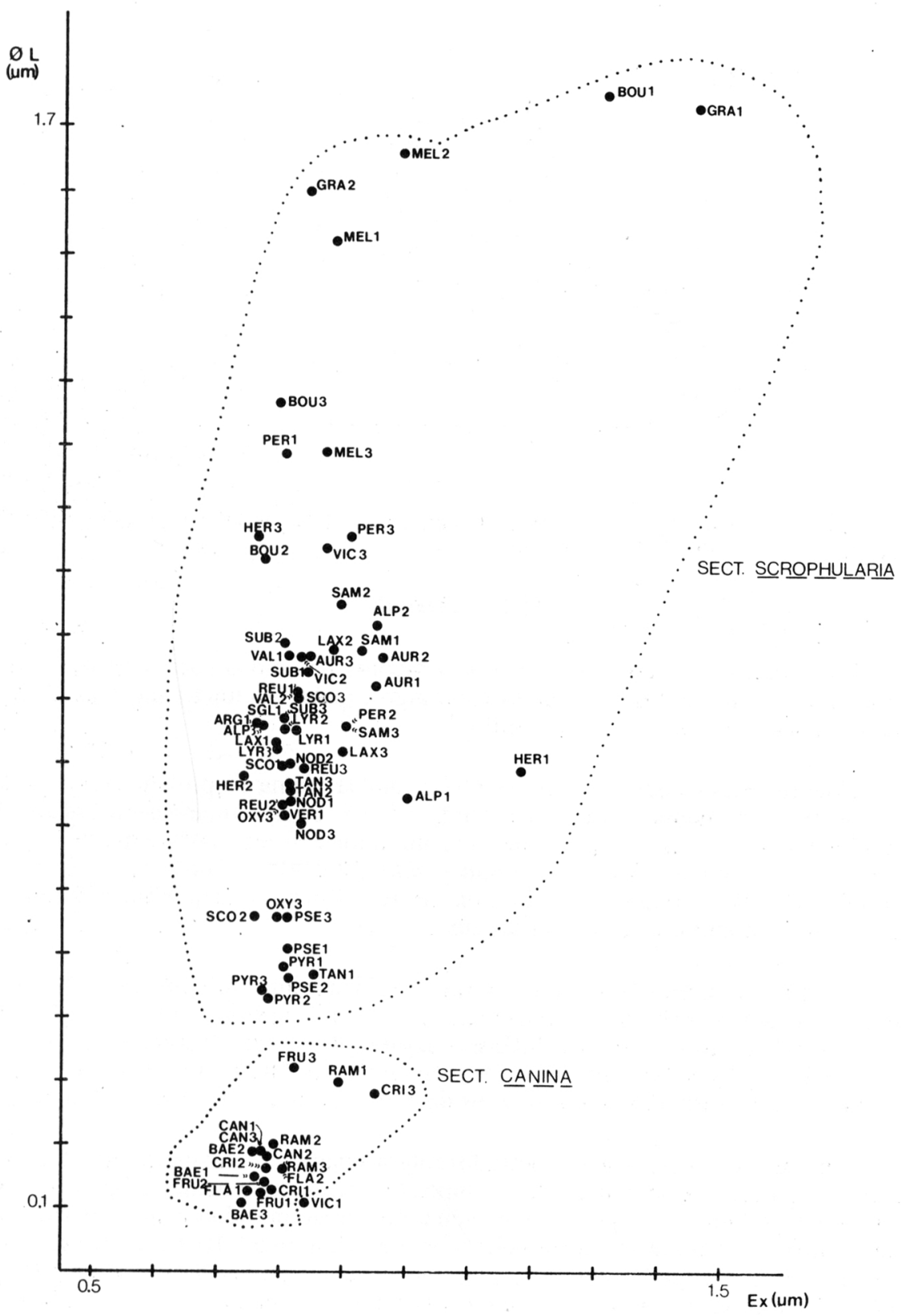

Fig. 2. Diagrama de dispersión simbólica de las poblaciones estudiadas del género Scrophularia en la Península Ibérica e Islas Baleares en función del diámetro de los lúmenes (L) y de la anchura de la exina (Ex) en los granos de polen. 


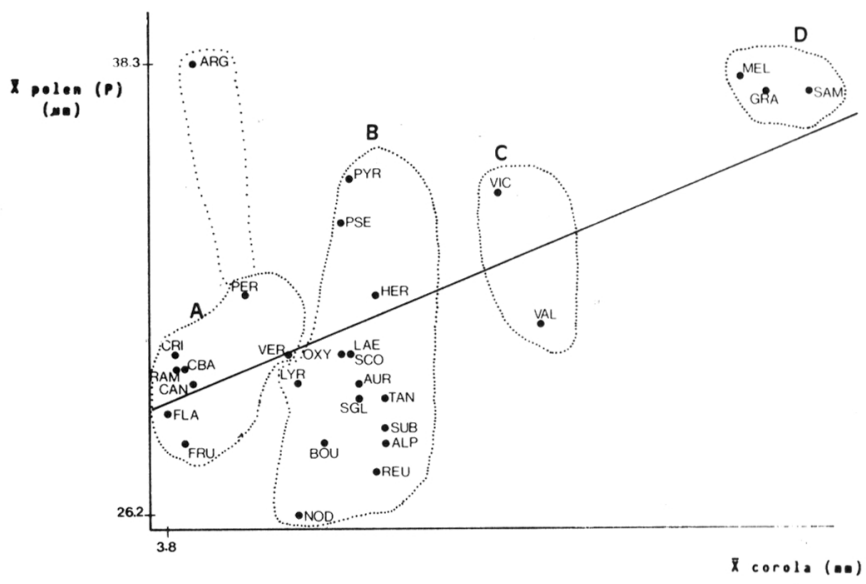

Fig. 3. Recta de regresión entre los valores del tamaño medio del polen $(\mathrm{P})$ y de la corola. (Nivel de significación: $* * * 99.9 \%$ de seguridad).

\section{RESULTADOS}

En la tabla 1 se exponen los valores medios obtenidos para los parámetros estudiados y el valor de $\mathrm{P} / \mathrm{E}$, indicándose el rango y la desviación típica $(\sigma)$ así como el intervalo de confianza (I.con.) para P y E.

Forma. Polen isopolar y con simetría radial (Lámina 1), prolado, subprolado o menos frecuentemente prolado-esferoidal (3,9\% de las muestras estudiadas), predominantemente longiaxo por presentar un valor P/E que varía entre 1,1 y 1,4 (véase Tabla 1), siendo mínimo en S.bourgeana (BOU-3), S.frutescens (FRU-1) y S.reuteri (REU-2), y máximo en S.valentina (PSE-1). En visión ecuatorial es elípticocircular y en visión polar, circular-triangular.

Tamaño. Polen variable tanto intraespecífica como intrapoblacionalmente, mediano, con eje polar (P) que oscila entre $25,4 \mu \mathrm{m}$ en S.nodosa (NOD-2) y 42,1 $\mu \mathrm{m}$ en S.sambucifolia subsp.mellifera (MELL-1), y eje ecuatorial (E) comprendido entre $19,1 \mu \mathrm{m}$ (S.nodosa, NOD-2) y 29,8 um (S.grandiflora, GRA-2). Los valores de la apocolpia varían entre 3,6 y $6,9 \mu \mathrm{m}$, y los de la mesocolpia entre 14,6 y 25,6 $\mu \mathrm{m}$.

Aperturas. Polen 3-zonocolporado (colporoidado) y angulaperturado (Lámina 1), con ectoaperturas terminales de tipo colpo con una anchura media máxima de 3,4$5,9 \mu \mathrm{m}$ (en visión polar), y que presentan en corte óptico meridiano una constricción o puente a nivel ecuatorial a modo de depresión (véase Lámina 1, B). Las endoaperturas son de tipo poro, lalongadas (Lámina 1) y con una anchura media de 1,9-6 $\mu \mathrm{m}$, a veces de muy difícil observación. La membrana apertural es granulada.

Exina. Presenta un grosor medio de 0,7-1,5 $\mu \mathrm{m}$ en la mesocolpia, con sexina aproximadamente de igual grosor que la nexina, tectum parcial e infratectum columelado (Lámina 1, F). 


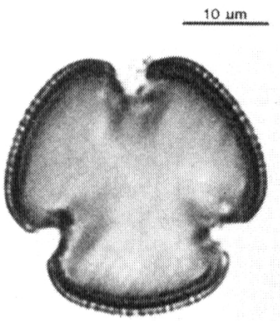

A
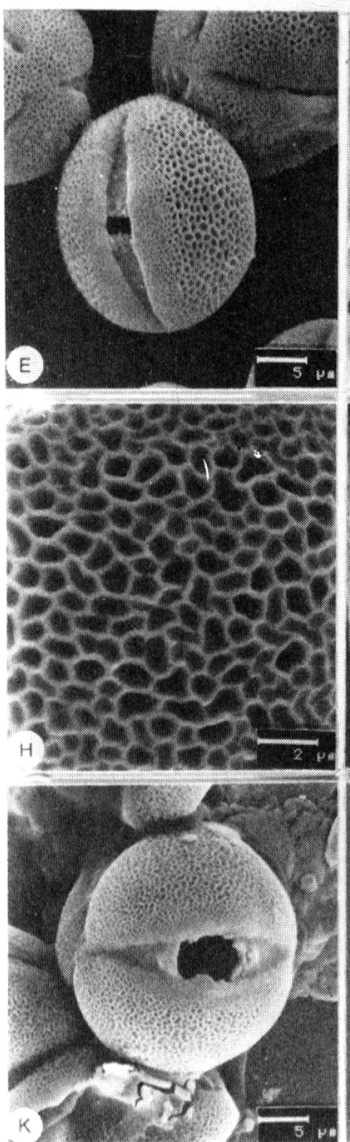
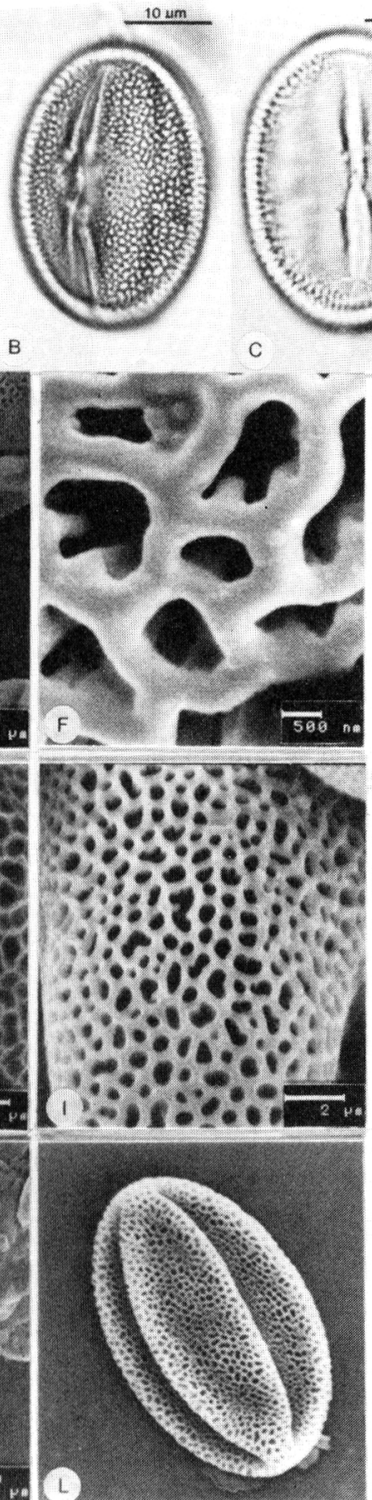
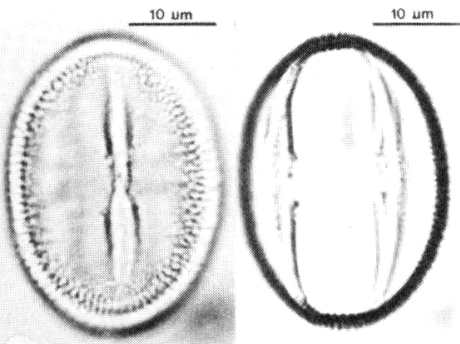

D
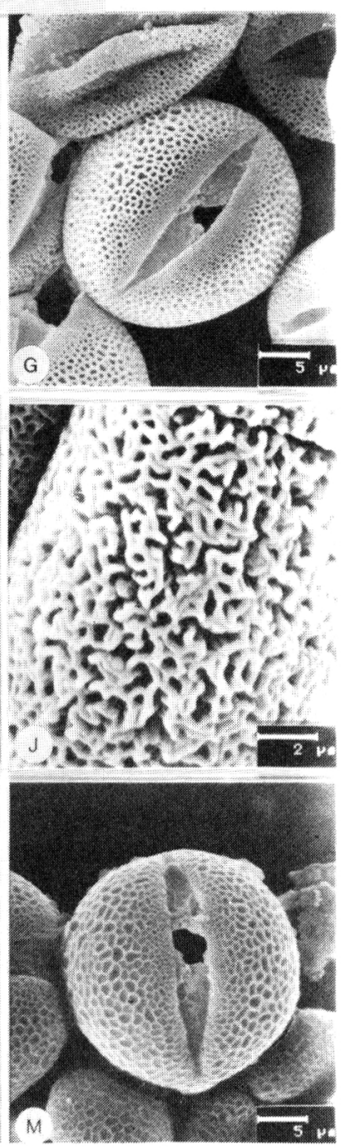

Lámina 1. Aspectos del polen en el género Scrophularia a microscopía óptica (A-D) y electrónica de barrido (E-M). A, S.sublyrata (SUB-3), visión polar. B-C, S.viciosoi (VIC-3), visión ecuatorial. D, S.valdesii (VAL-3), visión ecuatorial. E, S.tanacetifolia (TAN-1), visión ecuatorial. F, S.oxyrhyncha (OXY-1), detalle del retículo. G, S.valdesii (VAL-2), visión ecuatorial. H, S. peregrina (PER-1), detalle del retículo. G, S. valdesii (VAL-2), visión ecuatorial. $\mathrm{H}, \mathrm{S}$. pergrina (PER-1), detalle del retículo. I, S. oxyrhyncha (OXY-1), detalle del retículo. J, S. viciosoi (VIC-1), detalle del retículo irregular. C, S. crithmifolia 9CRI-3), visión ecuatorial. L, S. oxyrhyncha (OXY-1), visión ecuatorial. M, S. bourgeana (BOU-3), visión ecuatorial.. 


\begin{tabular}{|c|c|c|c|c|c|c|c|c|c|}
\hline \multirow[t]{2}{*}{ Poblac. } & \multicolumn{3}{|c|}{$P$} & \multicolumn{3}{|c|}{ E } & \multirow[b]{2}{*}{$\mathrm{P} / \mathrm{E}$} & \multicolumn{2}{|l|}{ A } \\
\hline & Rango & $\bar{x}$ & I. con. & Rango & $\bar{x}$ & I. con. & & Rango & $\bar{x}$ \\
\hline LP 1 & $25,0-31,3$ & 27,96 & 0,86 & $17,5-22,5$ & 20,50 & 0,82 & 1,36 & $4,0-7,0$ & 5 , \\
\hline ALP 2 & $23,8-30,0$ & 26,51 & 0,80 & $17,5-25,0$ & & & & & \\
\hline ALP 3 & $27,3-33,2$ & 29,87 & 0,61 & $23,4-27,3$ & 25,19 & 0,52 & 1,19 & $3,2-5,6$ & 4,56 \\
\hline ARG 1 & & & 1,11 & $23,4-33,2$ & 26,93 & 1,12 & 1,42 & & 4,88 \\
\hline AUR 1 & $27,5-32,5$ & 30,71 & 0,70 & $20,0-26,3$ & 23,16 & 0,68 & 1,33 & & \\
\hline AUR 2 & $30,0-32,5$ & 31,01 & 0,54 & $22,5-26,3$ & 23,64 & 0,55 & 1,31 & $4,0-6,0$ & 5,30 \\
\hline AUR 3 & $25,4-31,2$ & 27,76 & 0,68 & & & 0,72 & 1,22 & $4,8-7,2$ & 6,00 \\
\hline BOU 1 & $27,5-37,5$ & 31,77 & 1,00 & $21,3-25,0$ & 23,02 & 0,44 & 1,38 & $4,0-7,0$ & 5,65 \\
\hline BOU 2 & $23,4-29,3$ & 26,94 & 0,92 & $17,6-25,4$ & 22,51 & 0,96 & 1,20 & $2,4-5,6$ & 3,88 \\
\hline $\mathrm{BOU} 3$ & $23,4-27,3$ & 25,77 & 0,47 & $21,5-26,3$ & 24,10 & 0,57 & 1,07 & $3,2-4,8$ & 3,92 \\
\hline CAN 1 & $27,3-31,2$ & 30,05 & 0,56 & $19,5-23,4$ & 21,23 & 0,50 & 1,42 & $4,0-5,6$ & 4,68 \\
\hline CAN 2 & $25,4-32,2$ & 29,52 & 0,72 & $20,5-23,4$ & 21,58 & 0,32 & 1,37 & $4,0-4,8$ & 4,60 \\
\hline CAN 3 & $25,4-33,2$ & 29,43 & 0,74 & $21,5-27,3$ & 23,71 & 0,56 & 1,24 & $4,0-5,6$ & 4,72 \\
\hline CBA 1 & $26,3-33,2$ & 30,29 & 0,80 & $19,5-24,4$ & 21,49 & 0,47 & 1,41 & $3,2-4,8$ & 4,12 \\
\hline CBA 2 & $27,3-35,1$ & 30 & 0,7 & $20,5-25,4$ & 22,88 & 0,5 & 1,35 & $3,2-4,8$ & 4,24 \\
\hline CBA 3 & $27,3-33,2$ & 29,12 & 0,62 & $17,6-23,4$ & 20,45 & 0,61 & 1,42 & $3,2-4,8$ & 3,76 \\
\hline CRI 1 & $29,3-39,0$ & 34,05 & 1,18 & $21,5-29,3$ & 25,13 & 0,81 & 1,36 & $5,6-9,6$ & 6,72 \\
\hline CRI 2 & $27,3-32,2$ & 30,20 & 0,77 & $19,5-25,4$ & 22,20 & 0,7 & 1, & $4,0-5,6$ & 5,22 \\
\hline CRI 3 & $25,4-29,3$ & 27,52 & 0,5 & $17,6-21,5$ & 19,19 & 0,69 & 1,43 & $3,2-5,6$ & 4,72 \\
\hline FRU 1 & $25,4-3$ & 29 & 0,8 & $23,4-29,3$ & 26,25 & 0,5 & 1,12 & $4,0-5,6$ & 5,00 \\
\hline FRU 2 & $24,4-29,3$ & 27,17 & 0,54 & $19,5-25,4$ & 23,27 & 0,6 & 1,18 & & 5,08 \\
\hline FRU 3 & $23,4-30,2$ & 28,16 & & $19,5-25,4$ & 22,78 & 0 & 1, & $4,0-5,6$ & 5,04 \\
\hline FLA 1 & $25,4-33,2$ & 29,96 & 0,86 & $19,5-24,4$ & 22,00 & 0,56 & 1, & & 4,88 \\
\hline FLA 2 & $25,4-30,2$ & 28,10 & 0,6 & $18,5-24,4$ & 20,68 & 0,75 & 1, & $3,2-4,8$ & 4,36 \\
\hline GRA 1 & $32,2-39,0$ & 35, & 0,8 & $25,4-31,2$ & 28,06 & $07-7$ & 1, & $5,6-8,0$ & 6,92 \\
\hline GRA 2 & $33,2-43,9$ & 39,61 & 1,32 & $27,3-33,2$ & 29,76 & 0,7 & 1,33 & $4,8-7,2$ & 6 \\
\hline HER 1 & $27,3-32,2$ & & 0 & $20,5-25,4$ & 23,47 & 0,6 & 1,26 & $4,0-6,4$ & 5,32 \\
\hline HER 2 & $29,3-36,1$ & 32,25 & 0,69 & $19,5-24,4$ & 22,40 & 0,6 & 1, & & 5 \\
\hline HER 3 & $31,2-35,1$ & 33,99 & 0,54 & $22,4-25,4$ & 24,05 & 0,4 & 1,4 & $4,0-6,4$ & 5,44 \\
\hline LAX 1 & 28,3 & 30 & & $21,5-25,4$ & 22,87 & 0, & 1, & & 5 \\
\hline LAX 2 & $27,3-33,2$ & 31,11 & 0,67 & $19,5-23,4$ & 21,58 & 0,4 & 1,44 & $4,0-5,6$ & 4,64 \\
\hline LAX 3 & $25,4-33,2$ & 29,18 & 0,98 & $17,6-23,4$ & 20,69 & 0,67 & 1,41 & $4,8-6,4$ & 5,52 \\
\hline LYR 1 & $26,3-33,2$ & 29,65 & 0,79 & $19,5-2$ & 89 & 0, & 1,42 & & 5,0 \\
\hline LYR 2 & $25,4-33,2$ & 29,12 & 0,79 & $20,5-25,4$ & 22,26 & 0,6 & 1, & $4,0-6,4$ & , \\
\hline LY & 29,3 & & & & 21,71 & 0, & 1, & & \\
\hline MEL 1 & $39,0-44,9$ & 42,14 & 0,63 & $27,3-31,2$ & 29,23 & 0,5 & 1,44 & & 6,28 \\
\hline MEL 2 & $31,2-37,1$ & 35,33 & & $254-332$ & 28,73 & 0,8 & 1,23 & $4,8-8,0$ & $33-2$ \\
\hline MEL 3 & $33,2-39,0$ & 36,38 & 0,72 & $23,4-2$ & 25,47 & 0 & 1,43 & & \\
\hline NOD 1 & $25,4-32,2$ & 27,37 & 0,82 & $19,5-23,4$ & 21,87 & 0,3 & 1,25 & $4,8-5,6$ & , \\
\hline NO & 2 & 25 & 0,71 & 17,6 & & $04 \quad-2$ & 1,33 & $4,8-6,4$ & \\
\hline NOD 3 & $23,4-28,3$ & 25,77 & 0,54 & $18,5-21,5$ & 19,85 & 0,3 & 1,3 & $4,8-6,4$ & \\
\hline OXY 1 & $29,3-37,1$ & 32,20 & 0,7 & $21,5-24,4$ & 23,21 & 0,2 & 1,39 & $4,8-6,4$ & \\
\hline OXY 2 & $27,3-31,2$ & 29,22 & 0,7 & 19, & 21,33 & 0,6 & 1, & & 5,1 \\
\hline OXY 3 & $27,3-3$ & 29,9 & 0,6 & $19,5-25,4$ & 22,40 & 0,5 & 1,34 & & $r$ \\
\hline PER 1 & ?o & 30 & 0 , & 22 , & 24,01 & 0,5 & 1,27 & $4,0-5,6$ & \\
\hline PER 2 & $30,2-33,2$ & 32,10 & 0,46 & $23,4-27,3$ & 24,99 & 0,4 & 1,29 & & 5, \\
\hline PER 3 & $29,3-35,1$ & 33,07 & 0,79 & $25,4-29,3$ & 27,08 & 0,60 & 1,22 & 4,8 & \\
\hline PSE 1 & $31,2-37,1$ & 33 & 0,6 & 21,5 & & 0 & 1,46 & & \\
\hline PSE 2 & $31,2-35,1$ & 33 , & 0,5 & $24,4-27,3$ & 25,77 & 0,4 & 1,3 & $4,8-6,4$ & \\
\hline PSE 3 & $31,2-3$ & 34 & 0,7 & $23,4-27,3$ & 26,05 & 0,5 & 1,3 & $4,8-6,4$ & \\
\hline PYR 1 & $31,2-37,1$ & 34,05 & 0,70 & $25,4-29,3$ & 27,07 & 0,7 & 1,26 & $4,8-6,4$ & \\
\hline PYR 2 & $33,2-39$ & 35 & 0,5 & $25,4-29,3$ & 27,56 & 0,5 & 1,30 & $5,6-7,2$ & \\
\hline PYR 3 & $30,2-3$ & 35 & 0,79 & $23,4-28,3$ & 26,77 & 0 & 1,3 & $4,8-7,2$ & \\
\hline RAM 1 & $27,3-3$ & 29,81 & 0,9 & $21,5-2$ & 23,72 & 0,7 & 1,2 & $3,2-6,4$ & A \\
\hline RAM 2 & $29,3-34,1$ & 31 & 0,56 & & 21,63 & 0,3 & 1,44 & $4,0-5,6$ & \\
\hline 13 & $27,3-3$ & 29 & 0,5 & $21,5-27,3$ & 24,75 & 0,64 & 1,20 & $4,8-6,4$ & \\
\hline REU 1 & $23,4-31$ & 26 , & 1,00 & $20,5-24,4$ & 22,44 & 0,44 & 1,2 & 5 & \\
\hline REU 2 & $24,4-27,3$ & 26,06 & 0,44 & $21,5-2$ & & 0,54 & 1,1 & $3,2-5,6$ & 4, \\
\hline REU 3 & $26,3-31,2$ & 29,41 & 0,64 & $19,5-25,4$ & 23,32 & 0,68 & 1,26 & $4,0-5,6$ & 4,7 \\
\hline
\end{tabular}




\begin{tabular}{llllllllll} 
SAM 1 & $37,1-48,8$ & 42,06 & 1,28 & $27,3-31,2$ & 29,13 & 0,58 & 1,44 & $4,8-6,4$ & 5,52 \\
SAM 2 & $33,2-38,0$ & 35,07 & 0,56 & $24,4-29,3$ & 26,35 & 0,55 & 1,33 & $6,4-8,0$ & 7,00 \\
SAM 3 & $33,2-40,0$ & 36,10 & 0,83 & $23,4-29,3$ & 25,03 & 0,78 & 1,44 & $6,4-7,2$ & 6,68 \\
SCO 1 & $27,3-33,2$ & 29,90 & 0,71 & $21,5-25,4$ & 23,91 & 0,42 & 1,25 & $4,8-6,4$ & 5,84 \\
SCO 2 & $29,3-35,1$ & 32,01 & 0,88 & $21,5-27,3$ & 24,05 & 0,75 & 1,33 & $4,8-6,4$ & 5,88 \\
SCO 3 & $27,3-31,2$ & 29,81 & 0,58 & $23,4-25,4$ & 24,45 & 0,42 & 1,22 & $5,6-6,4$ & 6,28 \\
SGL 1 & $25,4-32,2$ & 29,18 & 0,78 & $19,5-23,4$ & 21,46 & 0,70 & 1,36 & $5,6-6,4$ & 5,88 \\
SUB 1 & $23,4-30,2$ & 28,05 & 0,72 & $23,4-26,3$ & 24,50 & 0,41 & 1,15 & $4,8-7,2$ & 6,08 \\
SUB 2 & $25,4-31,2$ & 27,46 & 0,72 & $21,5-25,4$ & 23,23 & 0,60 & 1,18 & $5,6-6,4$ & 6,12 \\
SUB 3 & $28,3-31,2$ & 29,81 & 0,47 & $21,5-23,4$ & 22,82 & 0,31 & 1,31 & $4,8-7,2$ & 6,20 \\
TAN 1 & $27,3-33,2$ & 29,42 & 0,72 & $21,5-25,4$ & 22,74 & 0,49 & 1,29 & $4,8-6,4$ & 6,00 \\
TAN 2 & $26,3-33,2$ & 29,81 & 0,95 & $21,5-26,3$ & 23,07 & 0,5 & 1,29 & $5,6-6,4$ & 6,20 \\
TAN 3 & $27,3-29,3$ & 28,25 & 0,38 & $19,5-23,4$ & 22,33 & 0,52 & 1,27 & $4,8-6,4$ & 5,84 \\
VAL 1 & $29,3-34,1$ & 31,61 & 0,67 & $21,5-25,4$ & 23,47 & 0,61 & 1,35 & $4,8-6,4$ & 5,76 \\
VAL 2 & $29,3-33,2$ & 30,74 & 0,56 & $21,5-25,4$ & 23,36 & 0,58 & 1,32 & $5,6-7,2$ & 6,40 \\
VER 1 & $25,4-35,1$ & 29,86 & 1,00 & $22,4-27,3$ & 24,98 & 0,70 & 1,20 & $5,6-7,2$ & 6,08 \\
VIC 1 & $35,1-40,0$ & 37,45 & 0,61 & $26,3-31,2$ & 28,59 & 0,53 & 1,31 & $5,6-8,0$ & 6,92 \\
VIC 2 & $31,2-37,1$ & 34,43 & 0,72 & $25,4-31,2$ & 27,32 & 0,80 & 1,26 & $5,6-7,2$ & 6,60 \\
VIC 3 & $29,3-35,1$ & 32,27 & 0,74 & $23,4-29,3$ & 25,43 & 0,67 & 1,27 & $5,6-8,0$ & 6,20 \\
\hline
\end{tabular}

\begin{tabular}{|c|c|c|c|c|c|c|c|c|c|c|}
\hline \multirow[t]{2}{*}{ Poblac. } & \multicolumn{2}{|c|}{ M } & \multicolumn{2}{|c|}{ Ex } & \multicolumn{2}{|c|}{$\mathrm{C}$} & \multicolumn{2}{|c|}{$\operatorname{Pr}$} & \multicolumn{2}{|l|}{ L } \\
\hline & Rango & $\bar{x}$ & Rango & $\bar{x}$ & Rango & $\bar{x}$ & Rango & $\bar{x}$ & Rango & $\bar{x}$ \\
\hline ALP 1 & $12,0-17,0$ & 14,60 & $1,0-1,0$ & 1,00 & $3,0-5,0$ & 3,63 & $1,5-3,0$ & 2,40 & $0,60-1,00$ & 0,75 \\
\hline ALP 2 & $15,0-18,0$ & 16,65 & $0,8-1,0$ & 0,95 & $3,0-4,0$ & 3,65 & $2,0-5,0$ & 3,15 & $0,90-1,20$ & 1,02 \\
\hline ALP 3 & $17,6-20,8$ & 19,36 & $0,7-0,8$ & 0,77 & 3,2 & 4,32 & $2,4-4,0$ & 3,28 & $0,72-1,04$ & 0,86 \\
\hline ARG 1 & $08,4-21,6$ & 20,04 & $0,6-0,8$ & 0,76 & 3, & 4 & $2,4-4,8$ & 3,72 & $0,64-1,04$ & 0,86 \\
\hline AUR 1 & $14,0-18,0$ & 16,50 & $0,8-1,0$ & 0,95 & 3,0 & 3 , & $2,0-4,0$ & 3,35 & $0,80-1,00$ & 0,92 \\
\hline AUR 2 & $17,0-20,0$ & 18,25 & 0,9 - & 0,96 & $3,0-5,0$ & 3,95 & $3,0-6,0$ & 3,65 & $0,80-1,00$ & 0,97 \\
\hline AUR 3 & $16,0-20,0$ & 18,72 & 0,8 & 0,84 & $4,0-5,6$ & 4,72 & $2,4-4,0$ & 3,24 & $0,40-2,00$ & 0,97 \\
\hline BOU 1 & $15,0-18,0$ & 16,65 & 0,7 & 1,31 & $4,0-6,0$ & 4,80 & $1,0-3,0$ & 1,93 & $1,20-2,20$ & 1,86 \\
\hline BOU 2 & $14,4-17,6$ & 16,60 & 0,7 & 0,77 & $3,2-5,6$ & 4,56 & $1,6-4,0$ & 2,48 & $0,80-1,60$ & 1,12 \\
\hline BOU 3 & $16,0-17,6$ & 15,06 & $0,7-0,8$ & 0,79 & $3,2-4,8$ & 4,32 & $1,6-4,0$ & 2,88 & $1,04-1,60$ & 1,37 \\
\hline CAN 1 & $15,2-19,2$ & 17,52 & $0,7-0,8$ & 0,77 & $3,2-4,8$ & 4,36 & $2,4-4,0$ & 3,12 & $0,16-0,24$ & 0,19 \\
\hline CAN 2 & $15,2-17,6$ & 16,64 & $0,7-0,8$ & 0,78 & $3,2-4,8$ & 4,16 & $2,4-4,0$ & 3,20 & $0,08-0,24$ & 0,18 \\
\hline CAN 3 & $16,0-18,4$ & 17,56 & $0,7-0,8$ & 0,77 & $3,2-4,8$ & 4,24 & $3,2-4,8$ & 3,80 & $0,16-0,24$ & 0,19 \\
\hline CBA 1 & $14,4-17,6$ & 16,36 & $0,6-0,8$ & 0,76 & $2,4-4,8$ & 3,40 & $2,4-4,8$ & 3,40 & $0,08-0,24$ & 0,15 \\
\hline CBA 2 & $16,8-20,0$ & 17,96 & $0,7-0,8$ & 0,76 & $3,2-4,8$ & 4,04 & $2,4-4,8$ & 3,48 & $0,08-0,40$ & 0,19 \\
\hline CBA 3 & $14,4-18,4$ & 16,88 & $0,6-0,8$ & 0,74 & $3,2-4,8$ & 3,68 & $2,4-4,8$ & 3,44 & $0,08-0,16$ & 0,11 \\
\hline CRI I & $17,6-21,6$ & 19,08 & $0,6-0,9$ & 0,79 & $4,0-5,6$ & 4,76 & $3,2-5,6$ & 4,02 & $0,08-0,24$ & 0,13 \\
\hline CRI 2 & $16,0-19,2$ & 17,60 & $0,7-0,8$ & 0,78 & $3,2-4,8$ & 3,95 & $2,4-4,8$ & 3,68 & $0,08-0,40$ & 0,16 \\
\hline CRI 3 & $16,0-18,4$ & 17,12 & $0,7-0,8$ & 0,95 & $3,2-4,8$ & 4,44 & $2,4-5,6$ & 4,00 & $0,08-0,40$ & 0,28 \\
\hline FRU 1 & $16,8-19,2$ & 18,48 & $0,7-0,8$ & 0,77 & $3,2-4,8$ & 4,32 & $2,4-4,0$ & 3,40 & $0,08-0,16$ & 0,12 \\
\hline FRU 2 & $16,0-18,4$ & 16,96 & $0,7-0,9$ & 0,78 & $3,2-4,8$ & 4,12 & $3,2-4,8$ & 3,52 & $0,08-0,24$ & 0,14 \\
\hline FRU 3 & $14,4-18,4$ & 16,36 & $0,7-0,8$ & 0,82 & $3,2-4,8$ & 4,20 & $2,4-4,0$ & 3,64 & $0,08-0,40$ & 0,32 \\
\hline FLA 1 & $15,2-20,0$ & 18,08 & $0,7-0,8$ & 0,75 & $3,2-4,8$ & 4,24 & $2,4-4,8$ & 3,96 & $0,08-0,24$ & 0,13 \\
\hline FLA 2 & $14,4-20,0$ & 16,96 & $0,7-0,9$ & 0,79 & $3,2-4,8$ & 3,72 & $2,4-4,8$ & 3,16 & $0,08-0,40$ & 0,16 \\
\hline GRA 1 & $19,2-24,0$ & 22,04 & $1,2-2,0$ & 1,45 & $4,0-6,4$ & 5,08 & $3,2-5,6$ & 4,04 & $1,20-2,40$ & 1,84 \\
\hline GRA 2 & $20,8-25,6$ & 24,12 & $0,8-1,2$ & 0,84 & $4,8-6,4$ & 5,92 & $4,8-8,0$ & 6,00 & $0,80-2,40$ & 1,70 \\
\hline HER 1 & $16,0-19,2$ & 18,12 & $0,6-0,9$ & 0,79 & $3,2-4,8$ & 4,20 & $2,4-4,8$ & 3,60 & $0,64-1,60$ & 1,18 \\
\hline HER 2 & $17,6-20,0$ & 18,60 & $0,6-0,8$ & 0,74 & $4,0-4,8$ & 4,56 & $2,4-4,8$ & 3,76 & $0,40-1,20$ & 0,78 \\
\hline HER 3 & $18,4-20,8$ & 20,00 & $0,6-0,8$ & 0,76 & $4,0-4,8$ & 4,72 & $2,4-4,8$ & 3,53 & $0,80-1,60$ & 1,16 \\
\hline LAX 1 & $16,0-18,4$ & 17,60 & $0,7-0,8$ & 0,79 & $3,2-4,8$ & 4,40 & $2,4-5,6$ & 3,80 & $0,40-0,96$ & 0,83 \\
\hline LAX 2 & $16,0-18,4$ & 17,48 & $0,8-1,0$ & 0,88 & $4,0-4,8$ & 4,64 & $4,0-5,6$ & 4,64 & $0,64-1,60$ & 0,98 \\
\hline
\end{tabular}




\begin{tabular}{|c|c|c|c|c|c|c|c|c|c|c|}
\hline LAX 3 & 8,4 & 17,40 & $0,8-1,0$ & 0,90 & $3,2-5,6$ & 4,36 & $3,2-5,6$ & 4,52 & $0,64-1,04$ & 0,82 \\
\hline LYR 1 & $15,2-18,4$ & 16,88 & $0,7-1,0$ & 0,82 & $-4,8$ & 4,20 & $2,4-5,6$ & 4,12 & $0,64-1,44$ & 0,85 \\
\hline LYR 2 & $15,2-18,4$ & 17,08 & $0,7-0,9$ & 0,80 & $4,0-4,8$ & 4,36 & $2,4-4,8$ & 4,04 & $0,40-1,60$ & 0,84 \\
\hline LYR 3 & $16,0-19,2$ & 17,20 & $0,7-0,8$ & 0,79 & $3,2-4,8$ & 4,20 & $3,2-5,6$ & 4,16 & $0,40-1,20$ & 0,82 \\
\hline MEL 1 & $24,0-27,2$ & 25,56 & $0,7-1,2$ & 0,88 & $4,0-5,6$ & 5,00 & $3,2-6,4$ & 4,56 & $0,96-2,40$ & 1,62 \\
\hline MEL 2 & $18,4-23,2$ & 21,48 & $0,8-1,2$ & 0,98 & $4,0-4,8$ & 4,48 & $3,2-7,2$ & 4,80 & $1,20-2,40$ & ,76 \\
\hline MEL 3 & $19,2-22,4$ & 20,84 & $0,8-1,0$ & 0,86 & $4,0-5,6$ & 4,84 & $4,0-6,4$ & 5,44 & $0,80-2,00$ & 29 \\
\hline NOD & 15 & 16,84 & $0,8-0,9$ & 0,81 & 4,8 & 3,76 & $-4,8$ & 3,80 & $0,48-0,88$ & 0,74 \\
\hline NOD & 6,8 & 15,76 & $0,8-0,9$ & 0,81 & & 3,64 & 4,0 & 3,56 & $0,72-1,04$ &, 8 \\
\hline NOD & $15,2-17,6$ & 16,32 & $0,8-1,0$ & 0,83 &, 8 & 3,92 & $-4,8$ & 3,92 & $0,40-0,80$ &, 7 \\
\hline OXY 1 & $16,0-20,8$ & 18,16 & $0,8-0,8$ & 0,80 & 1,8 & 4,16 & $3,2-5,6$ & 4,44 & $-1,04$ & 8 \\
\hline OXY 2 & $16,0-20,0$ & 17,76 & $0,7-0,9$ & 0,80 & & 4,44 & 4,8 & 3,40 & 0,96 & 0 \\
\hline OXY & $16,0-18,4$ & 17,44 & $0,7-0,8$ & 0,79 & & 4,08 & 4,8 & 4,00 & 0,80 & 0 \\
\hline PER & $16,8-20,0$ & 18,52 & $0,8-0,9$ & 0,80 & & 4,0 & 4,8 & 4,04 & 1,84 & \\
\hline PER & $17,6-20,0$ & 18,92 & $0,8-1,0$ & 0,90 & & 4,72 & 4,8 & 4,04 & 1,20 & \\
\hline PER & $18,4-20,0$ & 19,64 & $-1,0$ & 0,91 & & 4,44 & & 4 & 1,60 & \\
\hline PSE & 18,4 & 19,00 & $0,8-0,9$ & 0,82 & & 4,44 & & & 80 & \\
\hline PSE 2 & 16,8 & 19,00 & 0 & 0,81 & & 4 & & 2 & 80 & \\
\hline PSE 3 & 19,2 & 19,68 & 0 , & 0,81 & & 4 & & 0 & 80 & \\
\hline PYR 1 & 19,2 & 20,4 & & 0,80 & & 4 & 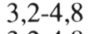 & 6 & 80 & \\
\hline PYR 2 & 20,0 & 21,64 & & 0 , & & & & 4 & 80 & \\
\hline PYR 3 & 19, & 20 & & 0 , & & & & 4 & 0,80 & 0,4 \\
\hline RAM 1 & 16 , & 1 & & 0 , & & 4 & & 88 & 0,40 & 1,3 \\
\hline RA & 18 & 1 & & 0 & & 8 & 1,8 & 3,80 & 0,24 &, 2 \\
\hline RA & 16 & 1 & 0,8 & 0 , & 8 & 4,12 & 4,8 & 4,28 & 0,32 & , 1 \\
\hline REL &, 2 & 17,52 & 0,9 & 0 , & 4,0 & 4,6 & $3,2-4,0$ & 3,40 & 1,20 &, 9 \\
\hline REU 2 & 8,4 & 17,64 & $0,8-0,9$ & 0,80 & 4,0 & 4,44 & $2,4-4,8$ & 3,48 & $-0,80$ & 0,7 \\
\hline REU 3 & $17,6-20,0$ & 18,80 & $0,8-1,0$ & 0 , & 4,0 & 4,4 & $3,2-4,0$ & 3,72 & 0,96 &, 1 \\
\hline SAM 1 & $20,8-24,8$ & 23,16 & $0,8-1,3$ & 0,92 & $4,8-5,6$ & 5,0 & $-8,0$ & 5,12 & 1,60 & 0,9 \\
\hline SAM 2 & $20,0-24,0$ & 21,60 & $0,8-1,0$ & 0, & 4,8 & 5,76 & $3,2-4,8$ & 3,92 & 1,60 & 1,1 \\
\hline SAM 3 & $20,8-24,0$ & 22,12 & $0,8-1,1$ & 0 , & 4,0 & 5,00 & $-4,8$ & 3,72 & ,60 & 0,8 \\
\hline $\mathrm{SCO}$ & $17,6-20,0$ & 18,76 & $0,8-0,9$ & 0 , & 4, & 4,32 & $-4,8$ & 3,80 & 1,04 &, 8 \\
\hline $\mathrm{SCO} 2$ & $18,4-20,8$ & 19,40 & $0,7-0,8$ & 0, & 3, & 4,44 & $3,2-4,8$ & 4,12 & 0,80 & 0,5 \\
\hline SCO 3 & $17,6-20,0$ & 18,72 & $0,8-1,0$ & 0, & $3,2-4,8$ & 4,12 & $-4,8$ & 3,60 & , 12 & 0,9 \\
\hline SGL & 16,8 & 18,32 & $0,8-0,8$ & 0 , & 4, & 4 & 4,0 & 3,60 & 04 & 0,8 \\
\hline SUB 1 & 17,6 & 19,20 & $0,8-0,9$ & 0 , & 8 & 4,60 & 4,0 & 52 & 28 & 0 \\
\hline SUB 2 & 16,8 & 18,20 & $0,8-0,8$ & 0 , & 8 & 3,96 & &, 72 & ,44 & 0,9 \\
\hline SUB 3 & 17,6 & 18,8 & 0,8 & 0 , & & 3,76 &, 6 & 4,36 & ,04 & ), \\
\hline TAN 1 & 16,8 & 18,40 & & 0,85 & 3,2 & 4,24 & & 3,84 &, 80 & 0,4 \\
\hline TAN 2 & $16,8-21,6$ & 18,52 & $0,8-1,0$ & 0,81 & & 4,32 & & 82 & ,04 & 0,7 \\
\hline TAN 3 & $16,8-19,2$ & 18,04 & $0,8-0,9$ & 0,81 & & 4,56 & & 72 & ,04 & 0,7 \\
\hline VAL 1 & $19,2-21,6$ & 20,28 & $0,8-0,9$ & 0,81 & & 4,60 & & 60 & $-1,44$ & 0, \\
\hline VAL 2 & $16,8-20,8$ & 18,84 & & 0,82 & & 4,36 & & 56 & 1,44 & 0,5 \\
\hline VER 1 & $17,6-22,4$ & 19,84 & $0,7-0,9$ & 0,80 & & 4,44 & & 4,08 & 0,80 & 0,7 \\
\hline VIC 1 & $19,2-24,0$ & 22,52 & $0,4-1,0$ & 0,84 & & 4,60 & & 4,60 & $-0,24$ & 0,1 \\
\hline VIC 2 & $19,2-22,4$ & 20,88 & $0,8-1,0$ & 0,83 & $4,0-5,6$ & 4,92 & $3,2-5,6$ & 4,20 & $0,80-1,36$ & 0,9 \\
\hline VIC 3 & $19,2-24,0$ & 20,56 & $0,8-1,0$ & 0,87 & $4,0-4,8$ & 4,48 & $3,2-4,0$ & 3,80 & $0,88-1,60$ & 1,1 \\
\hline
\end{tabular}

Tabla 1. Parámetros estudiados para cada población. P, longitud del eje polar en corte óptico meridiano. E, longitud del eje ecuatorial en corte óptico meridiano. A, longitud de la apocolpia en corte óptico ecuatorial. M, longitud de la mesocolpia en corte óptico ecuatorial. Ex, anchura de la exina en la mesocolpia. C, anchura del colpo en corte óptico ecuatorial. Pr, anchura del poro en corte óptico meridiano. $\mathrm{L}$, anchura de los lúmenes en la mesocolpia. Para cada uno de dichos parámetros se expresa el rango, la media $(\bar{x})$, la desviación típica $(\sigma)$ así como el intervalo de confianza para P y E. 


\begin{tabular}{llr}
\hline TAXA & $\begin{array}{l}\text { Tamaño medio } \\
\text { corola }(\mathrm{mm})\end{array}$ & $\begin{array}{r}\text { Tamaño medio } \\
\text { polen }(\mathrm{P}, \mu \mathrm{m})\end{array}$ \\
\hline
\end{tabular}

SECT. SCROPHULARIA

SUBSECT. SCROPHULARIA

\begin{tabular}{|c|c|c|}
\hline S. Nodosa & 6.25 & 26.19 \\
\hline S. bourgeana Lange in Willd. \& Lange & 6.85 & 28.16 \\
\hline S. herminii Hoffmanns. \& Link & 7.90 & 31.93 \\
\hline S. alpestris Gay ex Bentham in DC & 8.00 & 28.10 \\
\hline S. pyrenaica Bentham in DC & 7.25 & 35.04 \\
\hline S. auriculata Loefl. ex L. & 6.25 & 29.93 \\
\hline S. balbisii Hornem. subsp. balbisii & 7.50 & 29.83 \\
\hline \multicolumn{3}{|l|}{ S. balbisii Hornem. subsp. valentina } \\
\hline S. tanacetifolia Willd. & 8.00 & 29.16 \\
\hline S. scorodonia L. var. scorodonia & 7.25 & 30.57 \\
\hline S. scorodonia L. var. glabrescens Coutinho & 7.50 & 29.18 \\
\hline S. laxiflora Lange & 7.20 & 30.42 \\
\hline S. oxyrrhyncha Coincy & 6.20 & 30.44 \\
\hline S. sublyrata Brot. & 8.00 & 28.44 \\
\hline S. sambucifolia L. subsp. sambucifolia & 16.25 & 37.74 \\
\hline \multicolumn{3}{|l|}{ S. sambucifolia L. subsp. mellifera } \\
\hline (L’Hér. ex Aiton) Maire & 15.00 & 37.95 \\
\hline S. grandiflora DC. & 15.25 & 37.56 \\
\hline S. viciosoi Ortega Olivencia \& Devesa & 10.15 & 34.72 \\
\hline S. reuteri Daveau & 7.75 & 27.47 \\
\hline S. valdesii Ortega Olivencia \& Devesa & 11.00 & 31.18 \\
\hline S. peregrina $\mathrm{L}$. & 5.25 & 31.90 \\
\hline S. arguta Aiton & 4.30 & 38.29 \\
\hline \multicolumn{3}{|l|}{ SUBSECT. VERNALES Stiefelhagen } \\
\hline S. vernalis $\mathrm{L}$. & 5.65 & 29.86 \\
\hline \multicolumn{3}{|l|}{ SECT. CANINA G. Don } \\
\hline \multicolumn{3}{|l|}{ SUBSECT. CANINA } \\
\hline S. canina L. subsp. canina var. canina & 4.25 & 29.67 \\
\hline $\begin{array}{l}\text { S. canina } \text { L. } \\
\text { subsp. canina var. baetica Boiss. }\end{array}$ & 4.20 & 30.10 \\
\hline S. canina $\mathrm{L}$. . . & & \\
\hline subsp. ramasissima (Loisel.) Fourn. & 3.90 & 30.21 \\
\hline S. frutescens L. var. frutescens & 4.10 & 28.22 \\
\hline S. frutescens L. var. latifolia Bentham & 3.80 & 29.03 \\
\hline S. crithmifolia Boiss. & 4.00 & 30.59 \\
\hline
\end{tabular}

Tabla 2. Valores medios del tamaño de la corola y del polen (P) para cada uno de los táxones estudiados.

El tectum es en todos los casos reticulado, con lúmenes más anchos hacia la mesocolpia y menos hacia los colpos y apocolpia (Lámina 1, E y G). La anchura de los muros es variable, siendo mínima en $S$. peregrina (Lámina $1, H$ ) y $S$. bourgeana.

El retículo es más o menos regular en todos los táxones, salvo en la población VIC-1 de S.viciosoi en la que el polen mostró una notable irregularidad en los lúmenes y conformación de los muros (Lámina $1, \mathrm{~J}$ ), probablemente debido a una malformación o teratología de los granos de polen. La densidad del retículo varía según las especies, 


\begin{tabular}{l|lllllllll}
\multicolumn{1}{c}{} & $\mathrm{P}$ & $\mathrm{E}$ & $\mathrm{P} / \mathrm{E}$ & $\mathrm{A}$ & $\mathrm{M}$ & $\mathrm{Ex}$ & $\mathrm{C}$ & $\mathrm{PR}$ & $\mathrm{L}$ \\
\cline { 2 - 10 } $\mathrm{P}$ & 1.000 & & & & & & & & \\
$\mathrm{E}$ & $.796^{* * * *}$ & 1.000 & & & & & & & \\
$\mathrm{P} / \mathrm{E}$ & $.423^{*}$ & -.207 & 1.000 & & & & & & \\
$\mathrm{~A}$ & $.476^{*}$ & $.491^{*}$ & .032 & 1.000 & & & & & \\
$\mathrm{M}$ & $.864^{* * *}$ & $.848^{* * *}$ & .122 & .605 & 1.000 & & & & \\
$\mathrm{Ex}$ & .189 & .197 & .031 & .255 & .144 & 1.000 & & & \\
$\mathrm{C}$ & $.644 * *$ & $.642 * *$ & .079 & $.514 *$ & $.731^{* * *} .226$ & 1.000 & & \\
$\mathrm{PR}$ & $.588^{* *}$ & $.490^{*}$ & .022 & $.438^{*}$ & $.632^{* *}$ & -.075 & .422 & 1.000 & \\
$\mathrm{~L}$ & .276 & .358 & -.089 & .306 & .320 & $.548^{* *}$ & .422 & .130 & 1.000
\end{tabular}

Tabla 3. Correlaciones entre las 9 variables cuantitativas en los 29 táxones estudiados. (Nivel de significación: $* * * 99.9 \%$ de seguridad, $* * 99 \%$ de seguridad, $* 95 \%$ de seguridad).

siendo mayor en las especies de la Sect. Canina G.Don (0,1-0,3 $\mu \mathrm{m}$; S.canina, S.frutescens, S.crithmifolia) y menor en los táxones de la Sect.Scrophularia $(0,4-1,9$ $\mu \mathrm{m}$, resto de los táxones; Lámina $1, \mathrm{~K}-\mathrm{M})$; los mayores lúmenes los presentó S.bourgeana (Lámina 1, M).

\section{DISCUSIÓN}

Los caracteres polínicos analizados ponen de manifiesto el carácter estenopalino del género, por lo que en líneas generales resulta de escasa importancia taxonómica para la segregación de las especies, al menos en lo concerniente a la forma y al tamaño de los granos. Existe gran variabilidad en los valores de $\mathrm{P}$ y E, incluso intrapoblacionalmente. No obstante, táxones como S. arguta (ARG-1), S. grandiflora (GRA), S. sambucifolia subsp.sambucifolia (SAM) y S. sambucifolia subsp.mellifera (MEL) se segregan en general del resto por el valor de $\mathrm{P}$, pues presentan los granos de mayores dimensiones dentro del género.

De mayor interés es la correlación existente entre el tamaño del polen (expresado por P) y el tamaño de la corola (véase Fig.3, Tabla 2), lo que permite distinguir cuatro grupos. El primero (grupo A), que reúne los táxones que presentan corola muy pequeña $(x<5,7 \mathrm{~mm})$ y polen igualmente pequeño, lo integran todos los táxones de la Sect.Canina G.Don así como S. peregrina, S. arguta y S. vernalis (Sect.Scrophularia), todos ellos muy próximos a la recta de regresión, a excepción de $S$. arguta que se separa, contrariamente a lo esperado, por tener ejes polares de grandes dimensiones $(\mathrm{P}=38,3 \mu \mathrm{m})$.

El grupo $\mathrm{B}$ engloba las especies con corola mediana $(\mathrm{x}=6,2-8 \mathrm{~mm})$ y granos con $\mathrm{P}$ extremadamente variable, la mayoría de ellos bastante próximos a la recta de regresión. Por el contrario, el grupo $\mathrm{C}$ incluye a las especies de corola grande $(\mathrm{x}=8,1$ $12 \mathrm{~mm}$ ) y granos con $\mathrm{P}$ de $31,2-34,7 \mu \mathrm{m}$. Finalmente, en el grupo D se reúnen los táxones de corolas muy grandes $(x>15 \mathrm{~mm})$ y granos de grandes dimensiones $(\mathrm{P}>37,5$ $\mu \mathrm{m})$.

Es importante destacar que los táxones de la Sect.Canina se segregan del resto (a excepción de la población VIC-1 de S.viciosoi -Sect.Scrophularia-) en base a los exiguos lúmenes existentes a nivel de la mesocolpia (Fig. 2). Este carácter es de gran importancia taxonómica ya que condiciona la densidad del retículo, mayor en los granos de polen de las especies con número cromosómico bajo $(2 \mathrm{n}=24-26$; Sect. 
Canina G.Don), y menor en las que ostentan números cromosómicos altos ( $2 \mathrm{n} \geq 36$; Sect.Scrophularia; Ortega y Devesa, 1990 b). Asímismo, este carácter puede relacionarse también con la biología reproductiva de las especies, ya que los táxones de la sección Canina G.Don producen menos cantidad de granos de polen y de primordios seminales (Ortega, 1989) que los de la sección Scrophularia. Tal vez la mayor densidad del retículo polínico en la sección Canina G.Don sea una microadaptación morfológica de cara a la polinización que permita una mayor superficie de adherencia en los polinizadores, dado que además del menor número de granos de polen también producen menor cantidad de néctar (Ortega, l.c.) que los táxones de la sección Scrophularia y, por tanto, con esta adaptación del polen se asegure una menor pérdida de cargas polínicas en las visitas de los polinizadores.

No se ha observado ninguna correlación entre el número cromosómico y el tamaño polínico $(r=0,20 ; p>0,05)$.

Con objeto de discriminar algún tipo de segregación en base a los parámetros polínicos se efectuó un Análisis de Componentes Principales, no obteniéndose de éste información de interés para la delimitación de los táxones pues la mayoría de los parámetros estudiados ( 9 variables) están correlacionados entre sí (véase Tabla 3$)$. Así en dicha tabla se observa que existe una fuerte correlación entre las dimensiones de los ejes P y E, entre éstos y la mesocolpia (M), entre la mesocolpia y la anchura de los colpos (C), así como una correlación negativa entre E y P/E, P/E y L y entre Ex y Pr.

\section{Anexo 1. Procedencia de los táxones estudiados.}

S. alpestris Gay ex Bentham in DC. ALP 1 (HUESCA. Piedrafita, Peña Telera, JACA 573275); ALP2 (SORIA. Río Quesos, Santa Inés, MA 389461); ALP 3. (ASTURIAS. Puerto de Leitariegos, UNEX 6195).

S. arguta Aiton. ARG 1. (ALMERIA. Sierra de Gádor, P 89/66).

S. auriculata Loefl. ex L. LYR 1 (CACERES. Logrosán, UNEX 6052); LYR 2(SEVILLA. Entre Ecija y Herreram SEV 108461); LYR 3 (HUELVA. Arroyomolinos de León, SEV 101993). S. balbisii Hornem. subsp. balbisii. AUR 1 (NAVARRA. De Arraiz a Larrainzar, UNEX 6233); AUR 2 (BURGOS. Santalices, UNEX 6221); AUR 3 (ASTURIAS. Picos de Europa,, Tielve, UNEX 6213).

S. balbisii Hornem. subsp. valentina (Rouy) Ortega Olivencia \& Devesa. PSE 1 (TARRAGONA. Entre Duesaiguies y Ruidecanyes, UNEX 6016); PSE2 (BALEARES. Mallorca, entre Deia y Valdemosa); PSE 3 (CASTELLON DE LA PLANA. Font de la Barlasota, VAC 6744).

S. bourgeana Lange in Willk. \& Lange BOU 1 (SALAMANCA. Candelario, S de Béjar, MA 332851); BOU 2 (AVILA. Sierra de Gredos, MA 111294); BOU 3 (AVILA. Piedrafita. Pto. Peña Negra, MA 203491).

S. canina L. subsp. canina var. canina. CAN 1 (CIUDAD REAL. Villahermosa, SEV 98171); CAN 2 (ZAMORA. Perilla de Castro, SALAF 8620); CAN 3 (BADAJOZ. Llerena, UNEX 6112).

S. canina subsp. canina var. baetica Boiss. CBA 1 (ALICANTE. Elda, MA 375212); CBA 2, HUELVA. Desembocadura río Odiel, MA 389502); CBA 3 (SALAMANCA. Béjar, MA $111508)$.

S. canina subsp. ramosissima (Loisel.) Forn. RAM 1 (BALEARES. Menorca, El Grau, UNEX 6102); RAM 2 (BALEARES. Mallorca, Serra Nova, UNEX 6103); RAM 3 (BALEARES. Mallorca, Ca'n Picafort, SEV 41713).

S. crithmifolia Boiss. CRI 1 (LA RIOJA. Ribavellosa, SEV 52145); CRI 2 (MALAGA. Sierra Parda de Tolox, SEV 104067); CRI 3 (HUESCA. Balneario de Panticosa, UNEX 6161).

S. frutescens L. var. frutescens. FRU 1 (CADIZ. Entre Jerez y Puerto de Santa María, SEV 101663); FRU 2 (HUELVA. Matalascañas, SEV 97055); FRU 3 (LA CORUÑA. Noya, Playa del Testal, SEV 4762).

S. frutescens L. var. latifolia Bentham. FLA 1 (EXTREMADURA. De Charneca a Fonte 
de Telha, UNEX 6181); FLA 2 (BEIRA LITORAL. Entre Ponte da Varela e S. Jacinto, COI 931). S. grandiflora DC. GRA 1 (RIBATEJO. Coimbra, pr. a Beira, COI s.n.); GRA 2 (RIBATEJO. Coimbra, MA 111281).

S. herminii Hoffmanns. \& Link. HER 1 (ZAMORA. Sierra Segundera, MA 332849); HER 2 (ORENSE. Suacenza, S’ do Invernadeiro, MA 198186); HER 3 (LEON. Lago de la Baña, MA 292635).

S. laxiflora Lange. LAX 1 (CADIZ. El Jauto, UNEX 6204); LAX 2 (CADIZ. Los Barrios, UNEX 6207); LAX 3. CADIZ. Tarifa, Sierra del Niño, SEV 65016).

S. nodosa L. NOD 1 (LERIDA. Llivia, MA 111437); NOD 2 (ASTURIAS. De Arenas de Cabrales a Camarmeña); NOD 3 (ASTURIAS. Mestas de Con, UNEX 6188).

S. oxyrhyncha Coincy. OXY 1 (BADAJOZ. Puebla de Alcocer, UNEX 6144); OXY 2 (BADAJOZ. Puerto Mejoral, PCH 349); OXY 3 (BADAJOZ. Orellana de la Sierra, MA 415455).

S. peregrina L. PER 1 (GRANADA. Entre Almuñecar y Jete, UNEX 6168); PER 2 (BALEARES. Menorca. Torre de Visa Vell, UNEX 6169); PER 3 (BALEARES. Menorca, Pr. a San Cristobal, UNEX 6173).

S. pyrenaica Bentham in DC. PYR 1 (HUESCA. Bara, Cabeza de Guara, JACA 69982); PYR 2 (HUESCA. Mallos de Riglos, JACA 18977); PYR 3 (HUESCA. Rodellar, MGC 21934).

S. reuteri Daveau. REU 1 (AVILA. Navalonguilla, La Guilera, UNEX 5982); REU 2 (AVILA. Pradosiegar, MA 407237); REU 3 (AVILA. Entre Navalonguilla y Navalquijo, MA 332847).

S. sambucifolia L. subsp. sambucifolia. SAM 1 (RIBATEJO. Alcanede, MA 170049); SAM 2 (CADIZ. San Roque, MA 111271); SAM 3 (JAEN. Pasado Porcuna, UNEX 5986).

S. sambucifolia L. subsp. mellifera. MEL 1 (CADIZ. Entre Barbate y Vejer, UNEX 6021); MEL 2 (CADIZ. Chiclana, GDAC 23321); MEL 3 (SEVILLA. Entre Morón y Villanueva, UNEX 6206).

S. scorodonia L. var. scorodonia. SCO 1 (ASTURIAS. Entre Villaodrid y El Llano, JACA 204071); SCO 2 (MALAGA. Torcal de Antequera, UNEX 6075); SCO 3 SALAMANCA. Navasfrías, UNEX 6091).

S. scorodonia L. var. glabrescens (Coutinho) Ortega Olivencia \& Devesa. SGL 1 (BADAJOZ. Valle de Matamoros, UNEX 6096).

S. sublyrata Brot. SUB 1 (CACERES. Almorchón, PCH 2912); SUB 2 (CACERES. Valencia de Alcántara, UNEX 6148); SUB 3 (BADAJOZ. Alburquerque, UNEX 6149).

S. tanacetifolia Willd. TAN 1 (MURCIA. Sierra de Espuña, MA 415069); TAN 2 (ALMERIA. Antas, Pitón de Antas, MA 415084); TAN 3 (ALMERIA. Entre Macael y Tahal, SEV 26408).

S. valdesii Ortega Olivencia \& Devesa. VAL 1 (SALAMANCA. Pereña, MA 332848); VAL 2 (SALAMANCA. Presa de aldeadávila, UNEX 6023).

S. vernalis L. VER 1 (LA CORUÑA. Santiago, Meixon Frío, MAF 22695).

S. viciosoi Ortega Olivencia \& Devesa. VIC 1 (MALAGA. Torcal de Antequera, GDA 7202/13); VIC 2 (MALAGA. Alfarnate, MA 111441); VIC 3 (MALAGA. Ermita de Alfarnate, UNEX 6017).

AGRADECIMIENTOS. Los autores desean expresar su agradecimiento a la Dra. Ana Teresa Romero García, del Departamento de Biología Vegetal de la Universidad de Granada por la realización de las fotografías a microscopía electrónica de barrido en el Servicio Central de Microscopía de dicha universidad.

\section{BIBLIOGRAFÍA}

DALGAARD, V. -1979- Biosystematics of the Macaronesian species of Scrophularia. Op. Bot., 51: 3-64

ERDTMAN, G. -1943- An introduction to pollen Analysis. New York.

ERDTMAN, G. -1960- The acetolysis method -a revised description-. Svensk. Bot. Tidskr. ,54: 561-564.

ERDTMAN, G. -1969- Handbook of palynology. An introduction to the study of pollen and sporas. Copenhagen. 
ERDTMAN, G. -1971- Pollen morphology and plant taxonomy (Angiosperms). New York.

ERDTMAN, G., BERGLUND, B. \& PRAGLOWSKI, J. - 1961- An introduction to Scandinavian Pollen Flora. Scrophulariaceae. Grana Palynologica, 2(3): 56-57.

FAEGRI, K. \& J. IVERSEN -1975- Textbook of pollen analysis. Copenhagen.

GODOY, C. y M.J. DÍEZ - 1987- Scrophulariaceae. In: B.Valdés et al. (eds.). Atlas Palinológico de Andalucía Occidental, p. 302-303. Cádiz.

GRAU, J. -1979- Scrophularia. In: S.J.Mouterde (ed.). Nouvelle Flore du Liban et de la Syrie, 3: 235-241. Beyrouth.

GRAU, J. -1981- Scrophularia. In: K.H.Rechinger (ed.). Flora Iranica, 147: 213-284. Austria.

HASSALL, A.H. -1842- Observations on the structure of the pollen granule, considered principally with reference to its eligibility as a means of classification. Ann. Nat. Hist., 9: 544573.

HAYDEN, A. -1930- Structure and content of some pollen grains. Iowa Geol. Survey Bull., 7: $1001-1016$.

HIDEUX, M. -1972- Techniques d'étude du pollen au MEB: effects comparés des differents traitements physicochimiques. Micron, 3: 1-31.

INCEOGLU, O. \& F. KARAMUSTAFA -1977- The pollen morphology of plants in Ankara Region. IV. Scrophulariaceae. Communications Fac.Sci.Univ.Ankara (CZ:Bot), 21(8): 131144.

KOMAROV, V.L. -1955- Scrophularia. In: V.L.Komarov et al. (eds.). Flora U.R.S.S., 22: 229308. Leningrad.

LALL, S.S. \& R.R. MILL -1978- Scrophularia. In: P.H.Davis (ed.). Flora of Turkey, 6: 603-647. Edinburgh.

MOHL, H. -1835- Sur la structure et les formes des grains de pollen. Scrophulariaceae. Ann. Sci. Nat., ser.2, 3: 318-319.

MOORE, P.D. \& J.A. WEBB -1978- An illustrated guide to pollen analysis. London.

ORTEGA, A. -1989- Estudio taxonómico del género Scrophularia L. en la Península Ibérica e Islas Baleares. Tesis Doctoral (inédita). Universidad de Extremadura. Badajoz.

ORTEGA, A. Y J.A. DEVESA - 1990 a- Sobre la identidad de Scrophularia schousboei Lange y Scrophularia sublyrata Brot. Acta Bot.Malacitana, 15: 69-77.

ORTEGA, A. Y J.A.DEVESA -1990 b-Contribución al estudio cariológico del género Scrophularia (Scrophulariaceae) en la Península Ibérica e Islas Baleares. Lagascalia, 16(2): 171-198.

ORTEGA, A. Y J.A. DEVESA -1991- Dos táxones nuevos del género Scrophularia: S.viciosoi y S.valdesii. Candollea, 46: 111-118.

QAISER, M. -1982- Scrophularia. In: S.M.H. Jafri \& A.El-Gadi (eds.). Flora of Libya, 88: 2-9. Trípoli.

QAISER, M., S. KHATOON \& HAMIDULLAH -1988- The genus Scrophularia in Pakistan. Willdenowia, 17: 129-146.

PLA DALMAU, J.M. -1957-Estudios palinológicos y precisiones morfológicas sobre los granos de polen de quinientas especies botánicas del extremo N.E. de España. Tesis Doctoral, Univ. de Barcelona.

RISCH, C. - 1939- Die pollenkörner der in Deutschland wild wachsenden Scrophulariaceen. Ber. Deutsch. Bot. Ges., 57: 108-121.

SHAW, R.J. - 1962- The biosystematic of Scrophularia in western north America. Aliso, 5: 147-178.

STIEFELHAGEN, H. -1910- Systematische und pflanzengeographische Studien zur Kenntnis der Gattung Scrophularia. Vorarbeiten zu einer Monographie. Bot. Jahrb., 44: 406-496.

STRAKA, H. -1975- Pollenanalyse von Honig aus den Cañadas von Teneriffa (Kanarische Inseln). Decheniana, 127: 129-133.

VARGHESE, T.M. -1969- Studies in the family Scrophulariaceae. II Pollen morphology. Jour. Palyn., 4 (2): 91-97.

VERBEEK-REUVERS, A. -1976- On the number of endoapertures per ectoaperture in colporate pollen grains. Pollen et Spores, 18(3): 377-384.

(Aceptado para su publicación en Noviembre de 1991)

Dirección de los autores: Departamento de Biología y Producción Vegetal (Unidad de Botánica). Facultad de Ciencias. 06071 Badajoz. España. 